\title{
Massive first star binaries as new tools for Galactic archae- ology
}

\author{
Takuma Suda ${ }^{1, *}$, Takayuki R. Saitoh ${ }^{2,}$,Yuki Moritani ${ }^{3}$, Tadafumi Matsuno ${ }^{4}$, and Toshikazu \\ Shigeyama ${ }^{5}$ \\ ${ }^{1}$ Department of Liberal Arts, Tokyo University of Technology, Tokyo 144-8535, Japan \\ ${ }^{2}$ Department of Planetology, Graduate School of Science, Kobe University, Hyogo 657-8501, Japan \\ ${ }^{3}$ Kavli Institute for the Physics and Mathematics of the Universe (WPI), The University of Tokyo Insti- \\ tutes of Advanced Study, Tokyo 277-8583, Japan \\ ${ }^{4}$ Kapteyn Astronomical Institute, University of Groningen, 9747AD Groningen, the Netherlands \\ ${ }^{5}$ Research Center for the Early Universe, The University of Tokyo, Tokyo 113-0033, Japan
}

\begin{abstract}
Binary systems are important probes of the origin of stars with peculiar chemical features through the interactions between two stars. We have studied the evolution of a metal-free massive binary after the collision of the supernova ejecta with a low-mass companion. Theoretical models are developed using simulations of binaries after core-collapse supernovae using stellar evolution models, supernova ejecta models, and hydrodynamic simulations of the system consisting of supernova ejecta and companion stars. We find that these first star survivors will be observed as metal-rich halo stars in our Galaxy. In combination with the theoretical research, we looked for observational counterparts in the Galactic halo using the observational database where elemental abundances and kinematic data are available. We have also searched for the progenitor binary systems based on radial velocity monitoring. We report the current status of the search for massive binaries in the solar vicinity. The proposed scenario demands a new channel of star formation in the early universe and is a supplementary scenario for the origin of the known metal-poor stars.
\end{abstract}

\section{Introduction}

The search for the first stars in the universe is one of the important goals in astronomy. We proposed a new scenario to identify the survivors of the first stars in the Galactic halo [1]. Our scenario assumes a binary system consisting of massive stars and low-mass stars in a short distance so that the supernova explosion of the primary stars can influence the surface of the companion star at the end of the life of the massive primary. Figure 1 shows the schematic picture of our proposed massive binary scenario.

We performed simulations to quantify the impact of stripping from and accretion onto the surface of companion stars when the ejecta of massive primary collide with the low-mass companion. We also looked for evidence of observational counterparts in the Galaxy through the survey of massive binaries in the solar vicinity using the medium-resolution spectrograph.

\footnotetext{
*e-mail: sudatkm@stf.teu.ac.jp
} 
In this paper, we review the results of our simulation to provide an example of the survivor of the first stars with the massive binary scenario. The current status of the binary star survey is also reported.

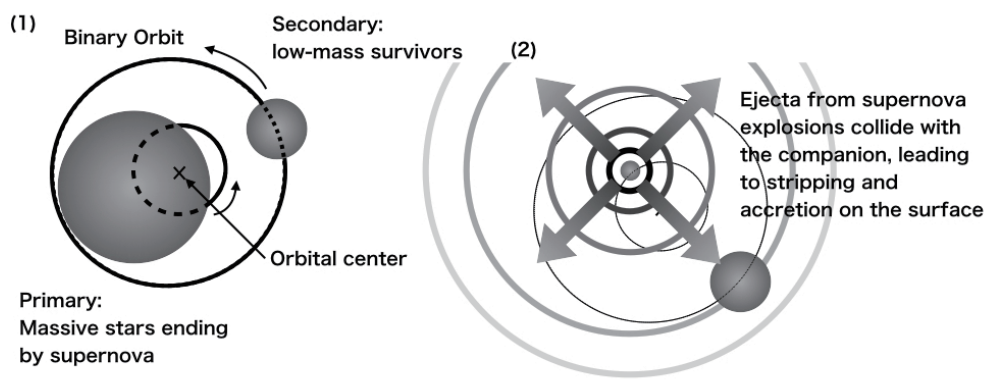

Figure 1. Schematic picture of our massive binary scenario to predict the survivors of the first stars in the Galactic halo.

\section{Simulation of the massive binary scenario}

Stellar models are taken from a primordial $0.8 M_{\odot}$ star [2] and the first supernova of a $15 M_{\odot}$ star [3]. They are mapped on the 3D space to perform SPH simulations using the ASURA code [4]. We followed the evolution of the binary system after the explosion of the primary star by tracing the SPH particles that collide with the low-mass companion. The explosion model is based on the model of SN1987A [5] for simplicity. The detail of the simulation setup and feasibility study is provided in Suda et al. [1].

Figure 2 shows a snapshot of the simulation. We found that the stripping of the envelope by the ejecta does not have a strong impact on the surface chemical composition on the secondary star, while the impact of accretion, mainly caused by slower inner ejecta, is significant. Although depending on the degree of mixing of the ejecta before the collision, it is expected that the secondary star should be metal-rich if the separation is sufficiently small.

We predict that the survivors of the first stars that experienced the accretion of supernova ejecta can be found in the Galactic halo as very metal-rich stars. The comparisons of models with the observations are made with stellar lithium abundances using the SAGA database [6].

\section{Search for massive binaries}

We looked for metal-rich counterpart of the progenitors of the massive binary scenario because the first massive stars do not exist. We have conducted the survey to identify OB stars with low-mass companion in short-period binaries. The observations have been made by Medium And Low-dispersion Long-slit Spectrograph (MALLS) equipped to the Nayuta telescope and High Dispersion Echelle Spectrograph (HIDES) equipped to the telescope at the Subaru Telescope Okayama Branch Office. Figure 3 shows the ongoing analysis of a binary candidate, HD 93521. The details of the survey is described in Moritani et al. [7] and the result will be reported in a forthcoming paper.

\section{Acknowledgement}

This work is supported by JSPS Kakenhi Grant No. 16K05287. 


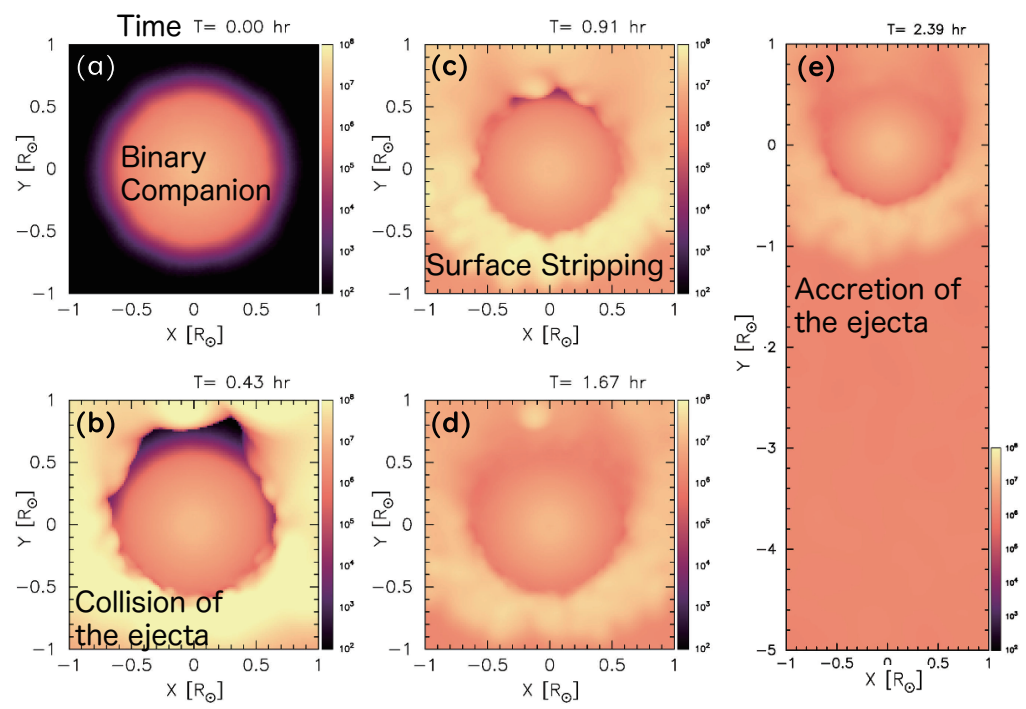

Figure 2. The snapshot of the SPH simulation of the collision between the supernova ejecta of a $15 M_{\odot}$ star and a $0.8 M_{\odot}$ star with a distance of $0.2 \mathrm{au}$. The color contour represents the temperature in units of kelvin. Panel (e) shows the simulation box that includes the part of the colliding ejecta.

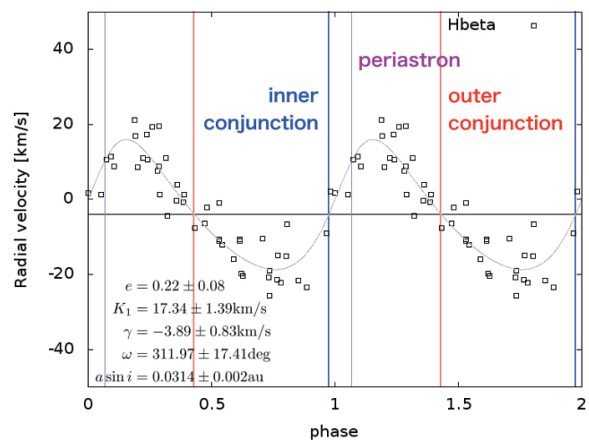

Figure 3. Determination of binary parameters for HD 93521. The derived parameters are given in the bottom left corner. The inner and outer conjuction, together with periastron are shown by the vertical lines.

\section{References}

[1] T. Suda, T. R. Saitoh, Y. Moritani, T. Matsuno, \& T. Shigeyama, PASJ 73, 609 (2021)

[2] T. Suda, \& M. Y. Fujimoto, MNRAS 405, 177 (2010)

[3] A. Heger, \& S. E. Woosley, ApJ 724, 341 (2010)

[4] T. R. Saitoh, H. Daisaka, E. Kokubo, J. Makino, T. Okamoto, K. Tomisaka, K. Wada, \& N. Yoshida, PASJ, 60, 667 (2008)

[5] T. Shigeyama, \& K. Nomoto, ApJ, 360, 242 (1990)

[6] T. Suda, Y. Katsuta, S. Yamada, T. Suwa, C. Ishizuka, Y. Komiya, K. Sorai, M. Aikawa, \& M. Y. Fujimoto, PASJ, 60, 1159 (2008)

[7] Y. Moritani, T. Suda, T. Shigeyama, \& T. R. Saitoh, Stars and Galaxies 1, 1 (2018), arXiv e-prints, arXiv:1812.06583 